\title{
Enfermeira Estomaterapeuta no Cuidado a Pessoa com Colostomia
}

\author{
Stoma Therapist Nurse in Caring for the Person with Colostomy: \\ A Case Report \\ Karina Portugal ${ }^{1}$ \\ ${ }^{I}$ Serviço de Enfermagem do Hospital Santa Izabel; Salvador, Bahia, Brasil
}

Correspondence addresses:

Dra. Karina Portugal

karyportugal@hotmail.com

Received: September 26, 2019

Revised: October 23, 2019

Accepted: October 30, 2019

Published: December $2^{\text {nd }}, 2019$

Data Availability Statement: All relevant data are within the paper and its Supporting Information files.

Funding: This work was the result of author's initiative. There was no support of research or publication funds.

Competing interests: The author has declared that no competing interests exist.

Copyright

(C) 2019 by Santa Casa

de Misericórdia da Bahia.

All rights reserved.

ISSN: 2526-5563
A confeç̧ão do estoma ocorre por diversos motivos, sendo o mais frequente a neoplasia colorretal. A enfermeira estomaterapeuta atua desde a fase préoperatória, até o período pós-operatório, com aspectos preventivos, terapêuticos e de reabilitação. $O$ objetivo desse estudo é relatar um caso vivenciado por enfermeira especialista em estomaterapia, no cuidado ambulatorial a pessoa colostomizada e destacar as mudanças clínicas obtidas após acompanhamento especializado. Paciente com 84 anos com diagnóstico de neoplasia de pâncreas, apresenta fístula colo-vesical e indicação de colostomia. Primeira consulta assistencial com estomaterapeuta aconteceu após a alta hospitalar quando foi admitido no serviço de curativos especiais. É importante a participação da enfermeira estomaterapeuta na elaboração de um planejamento da assistência voltada para a prevenção das complicações e na realização de orientações adequadas, oferecendo ações de educação em saúde e de promoção do autocuidado para melhoria da qualidade de vida da pessoa com colostomia. Com este relato de caso observa-se que é imprescindível para os indivíduos submetidos à confecção de ostomias serem acompanhados em nível ambulatorial por uma equipe multidisciplinar abrangendo assistência da enfermeira estomaterapeuta. Palavras-chave: Estomia; Assistência Ambulatorial; Enfermagem.

The most frequent reason for stomatization is colorectal neoplasia. The stomatherapist nurse acts from the preoperative to the postoperative period, with preventive, therapeutic and rehabilitation aspects. The aim of this study is to report a case of a stomatherapist in the care of colostomized patient and to highlight the clinical changes obtained after this kind of specialized monitoring. A 84-year-old patient with a diagnosis of pancreatic neoplasia has a cervical fistula and an indication for colostomy. The first appoitment with stomatherapist happened after hospital discharge when the patient was admitted to the special dressing service. It is important the participation of the stomatherapist nurse in the elaboration of a care planning focused on the prevention of complications and the accomplishment of appropriate orientations, offering health education and self-care actions to improve the quality of life of the patient with colostomy. It is essential for individuals undergoing ostomy confection to be followed at an outpatient level by a multidisciplinary team, including assistance from the stomatherapist nurse.

Keywords: Ostomy; Ambulatory Care; Nursing.

\section{Introdução}

No Brasil, conforme estimativas do Instituto Nacional de Câncer, cerca de 17.380 casos novos de câncer de cólon e reto em homens e 
18.980 em mulheres serão diagnosticados cada ano do biênio 2018-2019. ${ }^{1}$ O ostoma ou estoma intestinal representa uma abertura originada de um processo cirúrgico, que permite a exteriorização da alça intestinal através da parede abdominal, com o intuito de eliminar efluentes, fezes e gases, que se depositam em um sistema coletor (bolsa coletora). A confecção do estoma intestinal ocorre por diversos motivos, sendo o mais frequente a neoplasia colorretal. Pode ser um processo temporário ou definitivo, conforme as condições e causas de sua confecção, podendo ser classificado em colostomia e ileostomia, mediante sua localização de confecção. O auxílio terapêutico das estomias, principalmente das colostomias aos distúrbios colorretais, são bastante consistentes, porém tal procedimento pode acarretar múltiplas complicações. As complicações geram hospitalizações mais longas e maiores taxas de readmissão, cursando com elevados custos hospitalares. ${ }^{2,3}$ A estomaterapia é uma especialidade exclusiva do enfermeiro, voltada para o cuidado de pessoas com estomias, feridas agudas e crônicas, fístulas, drenos, cateteres e incontinências anal e urinária. A enfermeira estomaterapeuta atua desde a fase pré-operatória até o período pós-operatório imediato, mediato, tardio e ambulatorial, com aspectos preventivos, terapêuticos e de reabilitação em busca da melhoria da qualidade de vida. ${ }^{4}$ Por ser importante o acompanhamento da enfermeira em todas as fases do processo cirúrgico, justifica-se a realização deste estudo, para melhora da percepção dos profissionais de enfermagem sobre cuidados ao paciente colostomizado em suas necessidades durante processo pré-operatório até o pós-operatório em destaque para a assistência ambulatorial.

\section{Objetivo}

O objetivo desse estudo é relatar um caso vivenciado por enfermeira especialista em estomaterapia, no cuidado ambulatorial a pessoa colostomizada e destacar as mudanças clínicas obtidas após acompanhamento especializado.

\section{Experiência de Caso}

Paciente com 84 anos, sexo masculino, aposentado, católico, com relato de evidência em tomografia computadorizada de abdômen, espessamento do sigmoide com íntimo contato com a parede da bexiga, após consulta com coloproctologista em dezembro de 2017. Após um mês, realizada retossigmoidoscopia flexível, identificou-se um pequeno pólipo retal sem outros achados, com seguimento clínico. Após oito meses, já com diagnóstico de neoplasia de pâncreas, paciente apresenta fístula colo-vesical com episódios de infecção do trato urinário de repetição e indicação de colostomia como conduta devido à idade do paciente e necessidade de iniciar quimioterapia para tratamento. Em outubro de 2018, foi realizada cirurgia para confecção de colostomia em alça de sigmoide por vídeo, sem intercorrências e programada seis sessões de quimioterapia. Paciente não teve consulta pré-operatória com estomaterapeuta para orientação e demarcação do local da confecção do estoma. A primeira consulta assistencial com estomaterapeuta aconteceu dois dias após a alta hospitalar quando foi admitido no serviço de curativos especiais referência no ambulatório de um hospital filantrópico, em Salvador, Bahia, Brasil. Durante avaliação admissional, o paciente encontrava-se ansioso, referindo algia e inseguro quanto aos cuidados com a colostomia. Observouse na consulta de enfermagem em estomaterapia complicação periestomal denominada dermatite por efluente devido ao corte inadequado da base adesiva do sistema coletor, edema da alça intestinal, resíduo de necrose de coagulação e muco serosanguinolento como características normais do pós-operatório, além de haste de sustentação, e estoma medindo $25 \times 35 \mathrm{~mm}$. O paciente recusou fotografia na primeira consulta, devido estado momentâneo de ansiedade e nervosismo. A conduta inicial foi limpeza da pele periestomal e estoma, com água e sabão 
líquido, utilização de sistema coletor drenável de duas peças na cor opaca com filtro de carvão integrado e base adesiva plana composta predominantemente de carboximetilcelulose com realização correta do corte da base adesiva e uso de protetor cutâneo spray sem álcool para proteger a pele periestomal do efluente. Em seguida, foi realizada orientação ao paciente e familiar sobre limpeza e troca do sistema coletor, demonstração dos diversos tipos de sistema coletor de acordo com marcas de diferentes laboratórios, prescrição de enfermagem do sistema coletor, protetor cutâneo, orientação de retorno em no máximo sete dias para reavaliação e encaminhamento para centro de referência de dispensação de sistema coletor. Paciente retornou para segunda consulta assistencial de enfermagem, mantendo edema do estoma (Figura 1) e medida de $25 \times 35 \mathrm{~mm}$, melhora parcial da dermatite por efluente. Foram realizados os cuidados de limpeza (água e sabão líquido) e troca do sistema coletor e uso de protetor cutâneo. Na terceira consulta houve melhora significativa da dermatite e redução do edema do estoma (Figuras 2A e 2B), paciente encontrava-se mais confiante para o cuidado ao estoma juntamente com a família. A quarta consulta assistencial foi o dia de retirada da haste plástica de sustentação, conforme solicitação médica e indicação da literatura, seguido de limpeza do estoma, medindo $25 \times 25 \mathrm{~mm}$, troca do dispositivo coletor e uso de protetor cutâneo (Figura 3). No retorno para quinta consulta, completando um mês após primeira consulta, foi realizada avaliação (Figura 4) em que a colostomia evolui com cor vermelho vivo brilhante, pele periestomal íntegra e produção de fezes pastosas. Paciente refere alimentação balanceada com ingesta hídrica adequada em acompanhamento nutricional e em condições de realizar o autocuidado em domicílio. Dada alta do serviço nesta consulta e reorientado quanto aos cuidados, identificação de possíveis complicações e retorno caso exista suspeita de complicação com estoma.

\section{Discussão}

Alguns diagnósticos de neoplasia possuem como modalidade de tratamento a intervenção cirúrgica e/ou quimioterapia, o que pode

Figura 1. Edema do estoma e medida de $25 \times 35 \mathrm{~mm}$.

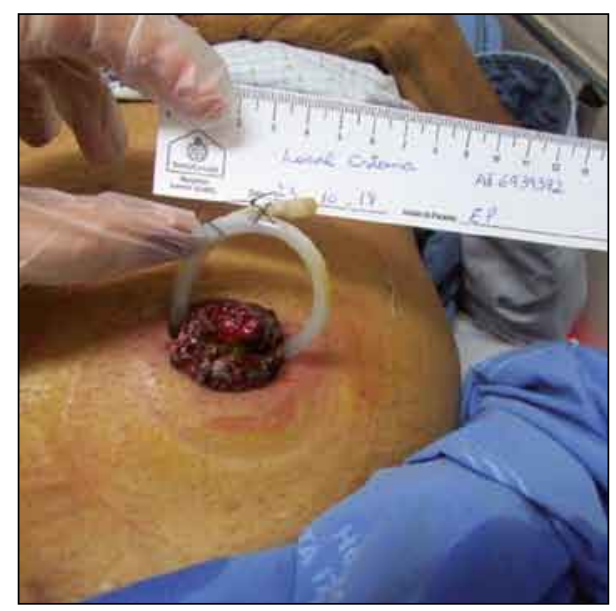

Figura 2. Redução do edema do estoma.
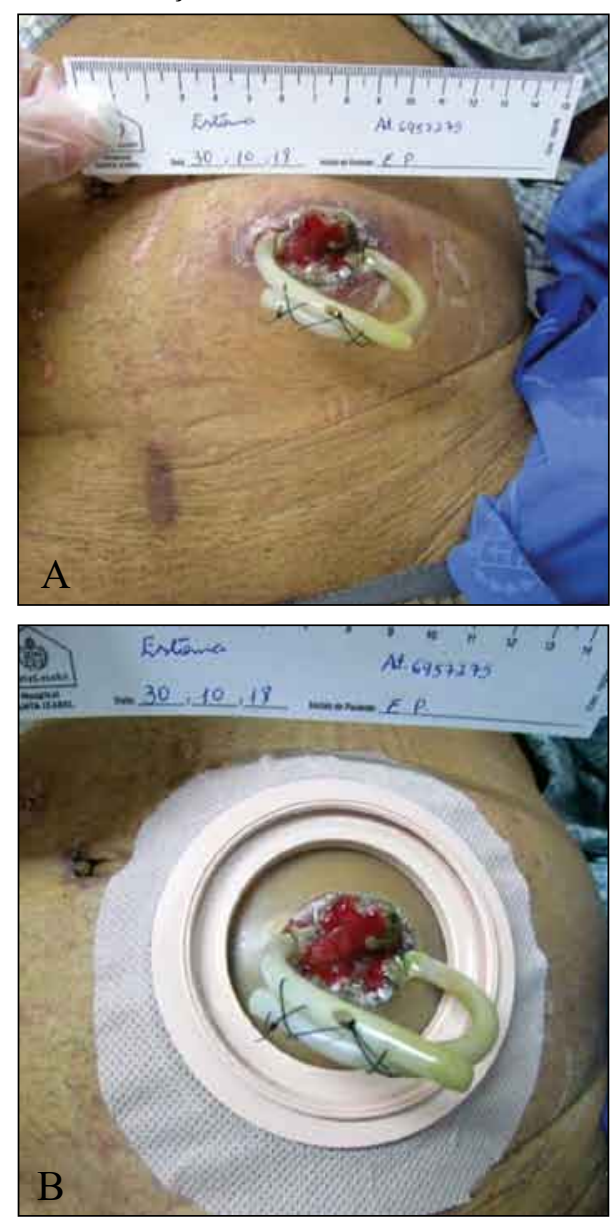
Figura 3. Retirada do retirada da haste plástica de sustentação, limpeza do estoma $(25 \times 25 \mathrm{~mm})$, troca do dispositivo coletor e uso de protetor cutâneo.

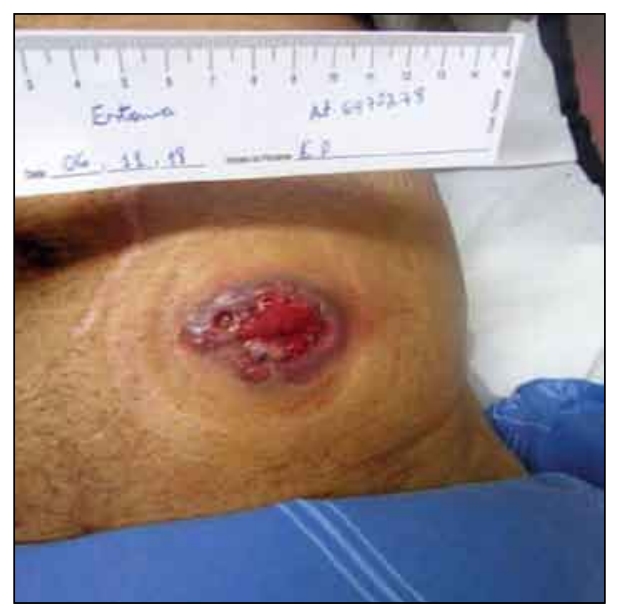

Figura 4. Colostomia com boa evolução, apresentando cor vermelho vivo brilhante, pele periestomal íntegra e produção de fezes pastosas.

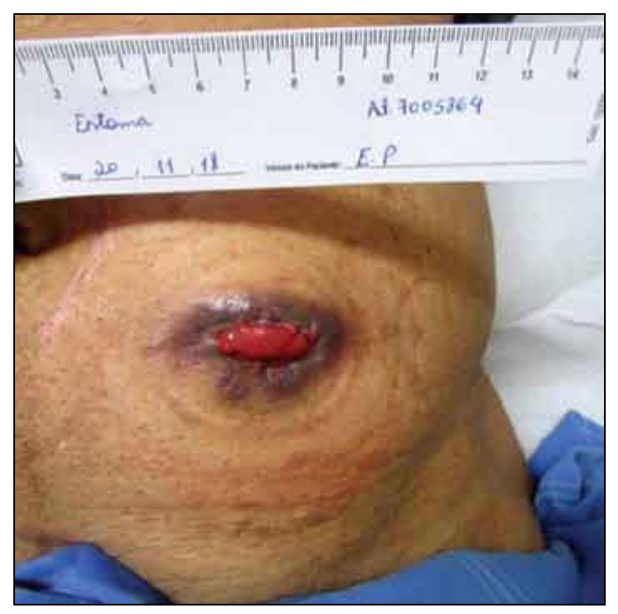

resultar na necessidade de confecção de ostomia intestinal. A colostomia gera algumas restrições corporais e mudanças nos hábitos de vida. Há questões de estética, limitações da movimentação corporal e há falta de controle nas eliminações de fezes e gases intestinais. $\mathrm{O}$ paciente necessita de atendimento sistematizado, especializado e multiprofissional para essas mudanças. As complicações em ostomias podem ser classificadas em recentes ou tardias. As complicações recentes abrangem, principalmente, dermatite em pele, retração ou necrose do estoma, desidratação. As tardias são, principalmente, hérnia paraestomal, prolapso estomal, estenose, fístula, ou abcesso periostomal. Pode haver ainda complicações a nível sistêmico, principalmente relacionadas a distúrbios hidroeletrolíticos em estomas de alto débito, anemia, pneumonia e sepse. No caso relatado, houve apenas uma complicação recente, dermatite por efluente, devido ao corte errado da base adesiva. ${ }^{3} \mathrm{Na}$ primeira consulta, longa e detalhada com duração em média de uma hora e meia,é indispensável que o individuo seja orientado sobre o autocuidado com o estoma, como instalar corretamente o sistema coletor, saber cortar a base adesiva na medida do estoma para proteger toda a pele ao redor do estoma, evitar vazamentos e lesões na pele periestomal. ${ }^{5-7}$ A portaria de $n^{\circ} 400 / 2009$, do Ministério da Saúde/Secretaria de Atenção à Saúde, assegura a necessidade de cuidados do paciente colostomizado em unidades de atenção básica e em serviços especializados, com estímulo ao autocuidado, promoção de saúde, prevenção de complicações, fornecimento de equipamentos coletores e adjuvantes e treinamento de profissionais de saúde..$^{3-6}$ Ressalta-se que compete ao enfermeiro especialista ou treinado para esse cuidado, intervenções exclusivas, visando ao atendimento especializado nas várias etapas do processo, com a melhor adaptação à condição do estomizado, promovendo o enfrentamento junto a família para melhor qualidade de vida do usuário..$^{4-9}$ É de responsabilidade do estomaterapeuta no período pré-operatório: consulta de enfermagem para obtenção de informações sobre paciente e família; orientar quanto ao ato operatório, ao estoma, equipamentos coletores, programas públicos de assistência, sobre declaração internacional dos direitos dos ostomizados e a associação de estomizados; além de fazer a demarcação do local onde será implantado o futuro estoma. No pós-operatório imediato e mediato avalia as condições do estoma e da ferida operatória, do efluente, a presença de complicações e condições do equipamento a fim de prescrever os cuidados e orientar a equipe; orientações de 
autocuidado ao paciente e cuidador. ${ }^{4}$ No pósoperatório tardio (ambulatorial ou domiciliar), a assistência ao colostomizado deve ser planejada junto com a equipe multiprofissional, conforme encaminhamento do cirurgião para enfermeira estomaterapeuta, assim é possível um atendimento especializado. Neste caso foram necessárias cinco consultas assistenciais com o paciente junto ao familiar no ambulatório e as principais intervenções da enfermeira estomaterapeuta foram avaliar as condições da pele periestomal, do estoma e presença de complicações; prescrever cuidados de estomaterapia; limpeza do estoma e troca do sistema coletor; estimular e/ou auxiliar o cuidado com colostomia reforçando as orientações. Após as intervenções da enfermeira estomaterapeuta notou-se melhora significativa da dermatite, paciente cursou mais confiante e aceitando o autocuidado. $^{4}$ Esse processo exige uma personalização das intervenções de enfermagem, ajustadas às necessidades específicas de cada pessoa. ${ }^{9} \mathrm{Na}$ Espanha, no estudo "Percepção de pacientes ostomizados sobre os cuidados de saúde recebidos", os participantes do estudo afirmaram que os enfermeiros estomaterapeutas são os especialistas em cuidados do estoma e que desempenham um papel chave em sua educação e informação. ${ }^{10}$ Diante do que foi exposto, na alta dos cuidados ambulatoriais especializados em estomaterapia o paciente, familiar ou cuidador deve dominar os cuidados, porém cada paciente deve ser visto de forma individualizada respeitando seu tempo de aceitação da nova condição. É de extrema importância a participação da enfermeira estomaterapeuta na elaboração de um planejamento da assistência voltado para a prevenção das complicações e na realização de orientações adequadas, dessa forma, a enfermeira passa a ser referência no cuidado para o estomizado, influenciando na reabilitação precoce, oferecendo ações de educação em saúde e de promoção do autocuidado para melhoria da qualidade de vida da pessoa com colostomia.,

\section{Conclusão}

Com este relato de caso observa-se que é imperioso para os indivíduos colostomizados, serem acompanhados pela enfermeira estomaterapeuta em todas as fases do processo cirúrgico em destaque no estudo para o pósoperatório tardio ambulatorial. Observandose a importância na atuação para prevenção de complicações, reabilitação precoce para as atividades diárias e autocuidado desses pacientes. Este estudo irá ampliar conteúdo teórico científico sobre esta temática para profissionais de enfermagem e área da saúde, para que estes façam direcionamento dos pacientes para continuidade da assistência ambulatorial com estomaterapeuta. Cabe salientar a necessidade da realização de novos estudos voltados para prática da enfermeira estomaterapeuta, a fim de fortalecer a atuação da categoria em suas atividades exclusivas.

\section{Referências}

1. Instituto Nacional do Câncer. Estimativa de Câncer no Brasil. INCA, 2018 ago 01 [acesso em: 2019 set 16]. Disponível em: <https://www.inca.gov.br/numeros-decancer>.

2. Medeiros LP, Silva IP, Lucena SKP, Sena JF, Mesquita EKS, Oliveira DMS, et al. Atividades da intervenção de enfermagem "cuidados com a ostomia". Rev enferm UFPE [Internet]. 2017 [acesso em 2019 set 12]; 11(Supl. 12):5417-26. Disponível em: <https:// periodicos.ufpe.br/revistas/revistaenfermagem/article/ viewFile/22899/25508>.

3. Oliveira IV, Silva MC, Silva EL, Freitas VF, Rodrigues FR, Caldeira LM. Cuidado e saúde em pacientes estomizados. Rev Bras Promoç Saúde [Internet]. 2018 [acesso em 2019 set 12]; 31(2): 1-9. Disponível em: $<$ https://periodicos.unifor.br/RBPS/article/view/7223>.

4. Yamada BA, Ferrola EC, Azevedo GR, Blanes L, Rogenski NM, Santos VLCG. Estomaterapia - competências do enfermeiro estomaterapeuta TiSobest ou do enfermeiro estomaterapeuta. Revista Estima [Internet]. 2019 [acesso em 2019 set 12]. Disponível em: <http://www.sobest. org.br/texto/1>.

5. Mota MS, Gomes GC, Petuco VM. Repercussões no processo de viver da pessoa com estoma. Texto Contexto Enferm [Internet]. 2016 [acesso em 2019 set 12]; 25(1):e1260014. Disponível em: <http://www.scielo.br/ $\mathrm{pdf} / \mathrm{tce} / \mathrm{v} 25 \mathrm{n} 1 / \mathrm{pt}$ 0104-0707-tce-25-01-1260014.pdf $>$. 
6. Silva ES, Castro DS, Garcia TR, Romero WG, Primo CC. Tecnologia do cuidado à pessoa com colostomia: diagnósticos e intervenções de enfermagem. Rev Min Enferm [Internet]. 2016 [acesso em 2019 set 12]; 20:e931. Disponível em: <http://www.reme.org.br/ artigo/detalhes/1065>.

7. Freitas RCL, Resende JL, Rodrigues PL, Magalhães MO, Carmo IC, Resende MA, et al. Importância da atuação do enfermeiro na demarcação do estoma no préoperatório mediato. Revista Eletrônica Acervo Saúde [Internet]. 2018 [acesso em 2019 set 12]; 11:S1567S1573. Disponível em: <https://www.acervosaude. com.br/doc/REAS173.pdf $>$.

8. Silva NM, Santos MA, Rosado SR, Galvão CM, Sonobe HM. Aspectos psicológicos de pacientes com estoma intestinal: revisão integrativa. Rev. LatinoAm. Enfermagem [Internet]. 2017 [acesso 2019 set 16]; 25: e2950. Disponível em: <http://www. scielo.br/scielo.php?script=sci_arttext\&pid=S0104- 11692017000100608\&lng=en. Epub 11 de dezembro de 2017. http://dx.doi.org/10.1590/15188345.2231.2950>.

9. Miranda LSG, Carvalho AAS, Paz EPA. Qualidade de vida da pessoa estomizada: relação com os cuidados prestados na consulta de enfermagem de estomaterapia. Esc Anna Nery [Internet]. 2018 [acesso em 2019 set 12]; 22(4):e20180075. Disponível em: <https:// repositorio- aberto.up.pt/bitstream/10216/53608/2/ Complicaes \%20das\%20ostomias\%20urinrias $\% 2$ $0 \mathrm{e} \% 20$ digestivas.pdf $>$.

10. Bonill-de las Nieves C, Capilla CD, Celdrán-Mañas M, Morales-Asencio JM, Hernández- Zambrano SM, Hueso-Montoro C. Percepção de pacientes ostomizados sobre os cuidados de saúde recebidos. Rev. Latino-Am. Enfermagem [Internet]. 2017 [acesso 2019 set 18];25:e2961. Disponível em: <http://www. scielo.br/pdf/rlae/v25/pt_0104-1169-rlae-25- e2961. pdf $>$. 